Review

\title{
Enzymatic Catalysis at Interfaces-Heterophase Systems as Substrates for Enzymatic Action
}

\section{Clemens K. Weiss and Katharina Landfester *}

Max Planck Institute for Polymer Research, Ackermannweg 10, 55128 Mainz, Germany;

E-Mail: weiss@mpip-mainz.mpg.de

* Author to whom correspondence should be addressed; E-Mail: landfester@mpip-mainz.mpg.de; Tel.: +49-6131-379-170; Fax: +49-6131-379-370.

Received: 18 January 2013; in revised form: 11 March 2013 / Accepted: 26 March 2013 /

Published: 9 April 2013

\begin{abstract}
Several important enzymatic reactions occurring in nature, such as, e.g., the digestion of fat, proceed only at the interface of two immiscible phases. Typically, these systems consist of an organic substrate, dispersed in an aqueous continuous phase, with a specialized enzyme capable of working at the interface. For adopting such a system for organic synthesis, a stable heterophase system with a large interfacial area is required. These prerequisites can be found in so-called miniemulsions. Such liquid-liquid heterophase systems feature droplets with sizes smaller than $500 \mathrm{~nm}$, and more importantly, these emulsions do not suffer from Ostwald ripening, as conventional emulsions do. Consequently, the droplets show long-term stability, even throughout reactions conducted in the droplets. In this review, we will briefly discuss the physicochemical background of miniemulsions, provide a comprehensive overview of the enzymatically catalyzed reactions conducted in miniemulsions and, as data are available, to compare the most important features to conventional systems, as reverse microemulsions, (macro)emulsions and solvent-based systems.
\end{abstract}

Keywords: enzymes; emulsion; miniemulsion

\section{Introduction}

The importance of biocatalytic processes and reactions for organic synthesis and the pharmaceutical, food and cosmetics industry has been constantly growing during the last few years $[1,2]$. From a synthetic 
point of view, enzymes are highly efficient catalysts for an extremely broad palette of reactions, ranging from esterification, over-oxidation and reduction to the formation of $\mathrm{C}-\mathrm{C}$ bonds [3]. Enzymes of one type, but from different origins, are specialized for substrates, positions in substrates and enantiomers. If the specialization is not enough, recent advances in understanding the genome and how to modify the genome of microorganisms fostered the controlled modification of individual amino acids in enzymes to generate highly specialized catalysts designed for a specific task [4,5]. Although the natural environment of enzymes is water and their preferred "natural" operation temperature is the temperature of the respective organism (typically $20-40{ }^{\circ} \mathrm{C}$ ), enzymes are very tolerant against temperature and organic solvents, especially, when the tertiary structure of enzymes is stabilized by immobilization on a solid substrate. Such immobilized enzymes also have the advantage of easy recovery from a product mixture, e.g., in an industrial process.

In recent years, the demand for "green" processes also increased, especially processes that are resource and energy saving and avoid components, such as heavy metals (conventional catalysts) or organic solvents. Several alternatives to organic solvents have been proposed, such as supercritical carbon dioxide or ionic liquids. The most favorable solvent, however, is water. It is inflammable, non-toxic, cheap and readily available. Moreover, transferring organic reactions from solvent-based systems to an aqueous environment may also affect conversion rates, as well as the regio- and stereo-specificity of certain reactions [6].

A huge number of organic compounds are, however, insoluble in water. Regarding liquid systems, emulsions result. Reactions between the organic phase and reactants dissolved in water may proceed at the interface or may be facilitated by phase transfer catalysts. In each case, the mass transfer is limited by the interface provided. Thus, methods to increase the interfacial area and additionally provide a stable reaction environment are of high interest. The requirements are met by so-called miniemulsions.

This review will give a brief introduction to the physicochemical features of miniemulsions, present a comprehensive overview over enzymatically catalyzed reactions conducted in miniemulsion, discussed in comparison to the respective conventional systems, as far as relevant data are available. We will conclude with some examples of miniemulsion-prepared nanostructures, which are responsive to the presence of an enzyme.

\section{Basic Features of Miniemulsions}

Imagine an equilibrated system consisting of $200 \mathrm{~mL}$ of soybean oil and $800 \mathrm{~mL}$ of water in a $1 \mathrm{~L}$ beaker with a diameter of $c a .15 \mathrm{~cm}$, the interfacial area is about $0.018 \mathrm{~m}^{2}$. Stirring this two phase system with a conventional magnetic stirring bar, an emulsion with droplet sizes of several $\mu \mathrm{m}$ is created. With an average diameter droplet size of $10 \mu \mathrm{m}$ (with $3.8 \times 10^{11}$ newly formed droplets), an interfacial area of $120 \mathrm{~m}^{2}$ results. Compared to the equilibrated system, this is a 6700 -fold increase of the interfacial area. Using techniques like ultrasound or high pressure homogenization, the droplet size can be reduced to several hundreds of $\mathrm{nm}$. The interface in the heterophase system increases again by a factor of 20, when the interface is reduced from $10 \mu \mathrm{m}$ to $500 \mathrm{~nm}$, providing ample surface for interfacial reactions or catalysis. However, the tendency of such very fine emulsions to degrade and regenerate the equilibrium state has to be counteracted. Droplet collision and subsequent coalescence can be suppressed by the addition of surfactants to the system. In addition to coalescence, diffusional 
degradation leads to the so-called Ostwald ripening. Curved surfaces, as present in small droplets, change the chemical potential of a component. The consequence is a pressure driving the material from the droplets to the surrounding phase (Laplace pressure). However, as well as the system is homogenized, the droplets do not have exactly equal diameters; there is a Laplace pressure gradient between the smaller and the larger droplets. Thus, there is a flux of matter from the smaller to the larger droplets. As a result, the smaller droplets disappear on account of the larger ones. The Laplace pressure can be counteracted by creating an osmotic pressure pointing to the opposite direction. This can be achieved by adding small amounts of a component to the system, which is soluble in the droplets (dispersed phase), but is the least soluble component in the surrounding phase (continuous phase). When both degradation mechanisms are controlled, the droplets are stable for a long time (in carefully prepared cases, at least several months). Such systems are typically referred to as "miniemulsions". The droplets have sizes below $500 \mathrm{~nm}$, and their size distribution is very narrow $[7,8]$.

In essence, the droplet in a miniemulsion can be regarded as an individual enclosed reaction space (a "nanoreactor"). Due to the absence of mass transfer, stoichiometric ratios of multiple components in the droplets do not change. Thus, a chemically and physically stable reaction environment can be provided, even when reactions are conducted in the droplets. If a solid product is created in the droplets, the material is directly formulated to a nanoscaled object. Thus, miniemulsions have mostly been used for the generation of functional and complex polymeric nanoparticles and capsules [8-10], as well as for the synthesis of inorganic nanoparticles [11]. However, the above mentioned features make the system excellently suited for interfacially catalyzed organic reactions. As direct miniemulsions are water-based systems, they are also qualified as environmentally benign and eco-friendly systems.

Although most of the reports about enzymatically catalyzed reactions make use of the huge interface in a miniemulsion, the enzyme can generally be located (1) in the disperse phase, (2) the continuous phase or (3) at the interface. To the best of our knowledge, no system with the enzyme located in the dispersed phase (referred to as (1)) has been published so far. The major system with an enzyme in the continuous phase (2) is the use of horseradish peroxidase (HRP) for radical polymerization. By far the most publications present work on enzymatic catalysis at the interface of miniemulsion droplets (3). These systems mostly make use of the interfacially active lipases or cutinase, which catalyze the formation or the cleavage of ester bonds between alcohols and carboxylic acids.

\section{Enzyme Located in the Disperse Phase}

Although a lot of systems with enzymes located in reverse micelles are known [12], to the best of our knowledge, such systems have not been realized in miniemulsion.

\section{Enzyme Located in the Continuous Phase}

The major system with the enzyme in the continuous phase is the HRP-initiated polymerization of styrene in miniemulsion. Derango et al. [13] first reported the possibility of using redox active enzymes for the generation of radicals, which can be used as the initiator for radical polymerization. Theses authors, as well as others, typically used water soluble monomers or mixtures of water and 
organic solvents to solubilize hydrophobic monomers, such as methyl methacrylate [14] or styrene [15]. HRP could, however, also be used for initiating styrene polymerization in emulsion. Shan et al. also used the initiator system of HPR, hydrogen peroxide $\left(\mathrm{H}_{2} \mathrm{O}_{2}\right)$ and a $\beta$-diketone, such as, e.g., pentanedione. They obtained, comparable to polystyrene emulsion polymerization with persulfate initiation, colloids with sizes of $80-100 \mathrm{~nm}$. The styrene conversion was high (>90\%); the polydispersity and molecular weights were comparable to those of the persulfate initiated reaction.

Figure 1. Catalytic cycle of horseradish peroxidase showing the possible mechanism for free radical polymerization of styrene (HRP, horseradish peroxidase; Ei, Eii and Eiii, oxidized states of horseradish peroxidase; $R, 2,4$-pentanedione radical). Reprinted with permission from [15]. Copyright (2000), American Chemical Society.

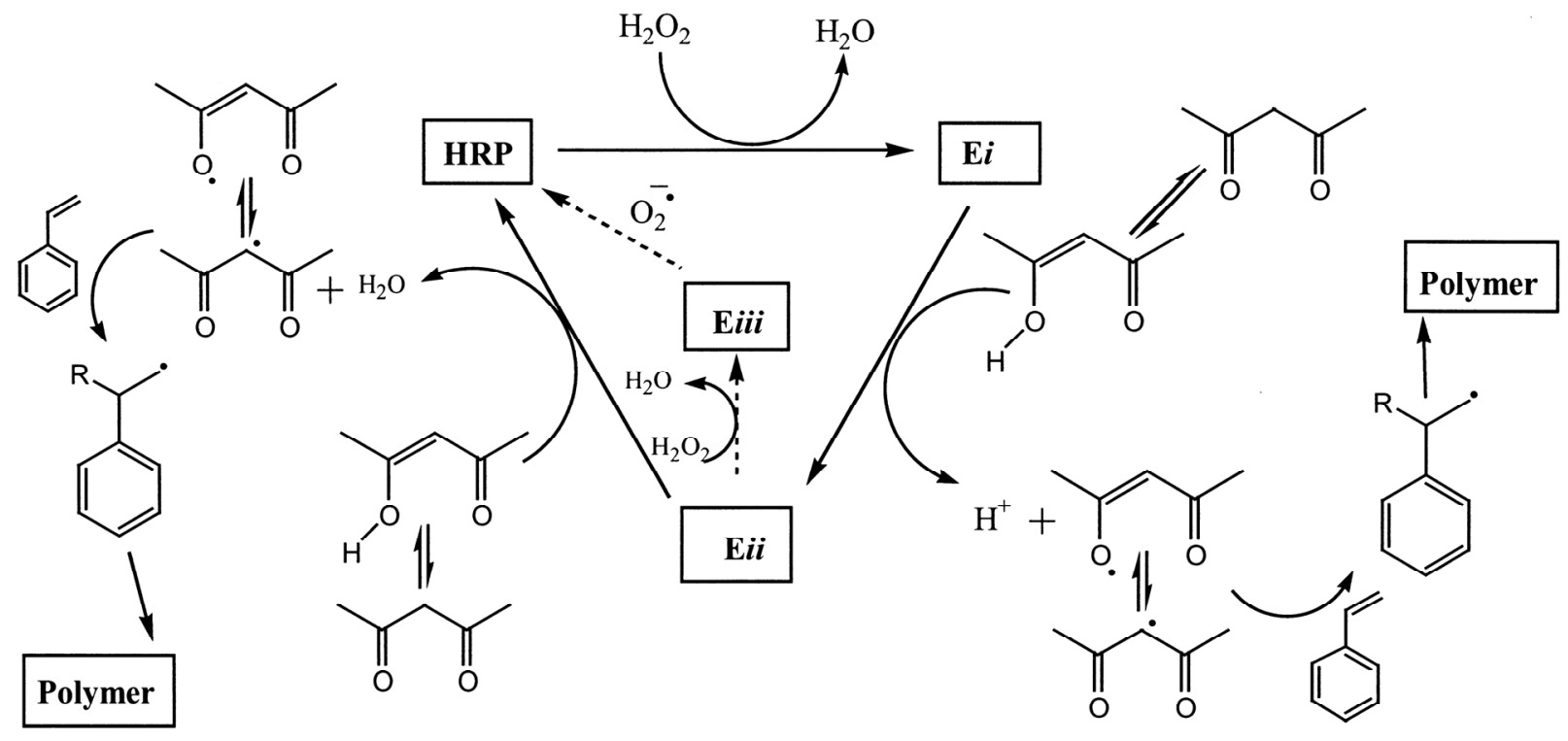

Qi et al. [16] performed the HRP-initiated styrene polymerization in miniemulsion using a protocol based on the optimum ratio of $\mathrm{H}_{2} \mathrm{O}_{2}$ and pentanedione [14]. The authors found that the time course of the monomer conversion was unlike that of conventional miniemulsion polymerization. In the beginning of the reaction, the conversion stayed at a constant conversion of $c a$. $8 \%$ for about $360 \mathrm{~min}$ to increase to $40 \%$ after $800 \mathrm{~min}$ and reach a final conversion of slightly below $50 \%$ after $24 \mathrm{~h}$. The decrease of the polymerization rate was potentially ascribed to a consumption of pentanedione and $\mathrm{H}_{2} \mathrm{O}_{2}$; the lag time in the beginning of the reaction was ascribed to a non-optimum ratio of pentanedione and $\mathrm{H}_{2} \mathrm{O}_{2}$. The optimal ratio was determined for a water/solvent system and not for an emulsion. This ratio is essential, as a misbalance may lead to oxidized HRP (Eiii, Figure 1), which is inactive. The pentanedione may partition between the aqueous and the styrene phase. Consequently, the ratio in the aqueous phase is not the intended one. Furthermore, there may be a competition between initiation with and without pentanedione [13], so that multiple mechanisms may act at the same time. The molecular weight was measured to be $M_{n}=406,000 \mathrm{~g} \mathrm{~mol}^{-1}$, which is higher than that $\left(M_{n}=30,000 \mathrm{~g} \mathrm{~mol}^{-1}\right)$ found in the water/solvent system with HRP initiation [15]. The authors found that the enzyme was quite sensitive to the temperature range. While at $23{ }^{\circ} \mathrm{C}$, almost $50 \%$ conversion was found; at $32{ }^{\circ} \mathrm{C}$, the conversion dropped to only $10 \%$. An inhibition or consumption of the enzyme 
is indicated by the fact that doubling the amount of the enzyme in the system led to an almost quantitative conversion.

A more in-depth study of that system was conducted by Kohri et al. [17,18]. These authors investigated the influence of the type of surfactant, the type of $\beta$-diketone, the ratio of pentanedione/ $\mathrm{H}_{2} \mathrm{O}_{2}$, the ratio of $\mathrm{H}_{2} \mathrm{O}_{2} / \mathrm{HRP}$ on the conversion of styrene, the molecular weight of the polymer and the activity of the enzyme. Sodium dodecyl sulfate (SDS, $10 \mathrm{mM}$ ) as the anionic surfactant has the least effect on the activity of HRP. The activity is $20 \%$ less than HRP activity in the absence of surfactant. The conversion of styrene in the miniemulsion, however, was only $13.5 \%$. This low conversion can be explained by the adsorption of HRP to the negative charges of the SDS-stabilized styrene droplets. Thus, the enzyme is denatured and loses its catalytic activity. Cationic or non-ionic surfactants, in contrast, significantly reduce the activity to about 50\% compared to HRP activity in the absence of surfactants. The conversion of styrene was found to be more than $70 \%$ and, in the case of a special cationic polymerizable surfactant, i.e., $N, N$-dimethyl- $N$ - $n$-dodecyl- $N$-2-methacryloyloxyethylammoniumbromide (C12-DMAEMA), almost 100\%. In the presence of sterically-stabilized droplets (non-ionic surfactants) or droplets stabilized with positive charges (cationic surfactants), HRP remains in solution and can catalyze the generation of pentanedione radicals, which can initiate styrene polymerization. The radicals can initiate styrene polymerization in aqueous solution, or the pentanedione radicals can enter the droplets and initiate polymerization there. The authors also found that the diketone has to have a sufficient water solubility to interact with the enzyme. Using the hydrophobic dibenzoylmethane, which is insoluble in water, no polymerization was detected. These results underline that the enzyme is located in the continuous aqueous phase of the miniemulsion and only acts here. Adsorption to the droplet interface presumably leads to denaturation of the enzyme, and consequently, the radical generation is no longer catalyzed.

This process is interesting, as it produces polymeric latexes with particles of around $100 \mathrm{~nm}$ at low temperatures. Typical miniemulsion with potassium persulfate initiation is conducted at $72{ }^{\circ} \mathrm{C}$ [19] and leads to comparable results. Droplet nucleation, which can be achieved when hydrophobic initiators, such as V59, are used, is not accessible with the HRP-mediated polymerization.

\section{Enzyme Located at the Interface}

In contrast to, e.g., HRP, which is deactivated upon adsorption to the interface between an organic and an aqueous phase, there are several known enzymes that can catalyze reactions at a liquid/liquid interface or even need the interface to be activated. Probably the most prominent examples for such enzymes are lipases [20-22]. Lipases are enzymes, which are found in a variety of organisms, from microorganisms to mammals. They belong to the group of hydrolases (E.C. 3.1.1.3) and are essential for fat digestion [23]. Thus, these enzymes need to act on water insoluble substrates. For that reason, lipases catalyze at the interface of hydrophobic droplets and water. In metabolism, their main task is to hydrolyze triacylglycerols (triglycerides, "fat" and "oil") into fatty acids and monoglycerides, which are resorbable for the organism. For organic synthesis, these enzymes are of great interest, as they can also catalyze the reverse reaction, i.e., the formation of an ester bond between a carboxylic acid and an alcohol under liberation of water (Scheme 1). 
Scheme 1. General reaction scheme for ester formation and hydrolysis.

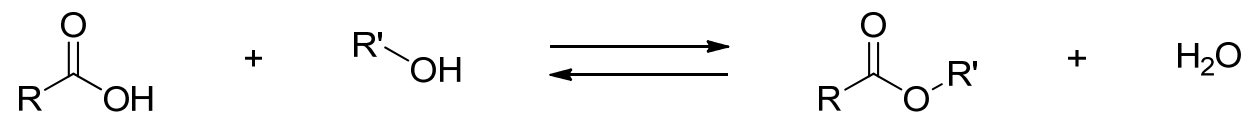

Lipases of different origins have a variety of preferences for catalyzing in the central or in the outer positions of glycerol (Scheme 2), have a preference for certain chain lengths of fatty acids [22] or catalyze reactions of primary and/or secondary alcohols [24]. In addition to the catalysis in "native" heterophase systems, i.e., oil-in-water emulsions, lipases have also shown their ability to work in reverse micelles (microemulsions) and in organic solvents [12,25-30]. Although some lipases are extremely tolerant to temperature, their preferred environment is at only slightly elevated temperatures.

Scheme 2. General structure of a triacylglycerol (triglyceride).<smiles>[R]C(=O)OC(C)=C(C)OC([R])=O</smiles>

Although it was well known that lipases are essential for fat hydrolysis and although there was evidence that enzyme preparations from pancreas [31,32] promote ester formation, the first systematic study on the use of lipases for the chemical synthesis of oleyl esters with a variety of alcohols was only published in the late 70s by Okumura et al. [24]. Lipases from four different organisms were evaluated for their ability to generate the respective esters. The experiments were conducted at $30{ }^{\circ} \mathrm{C}$ in aqueous emulsion with casein as the stabilizer, and depending on the lipase and the alcohol, conversions of up to $90 \%$ after $16 \mathrm{~h}$ were detected. Although the authors only esterified oleic acid with several diols, these results already suggested that also the polycondensation reaction may be accessible with lipase catalysis.

Despite the number of publications on lipases catalysis in emulsion, in reverse micelles and organic solvents, comparably few studies using the miniemulsion technique can be found in the literature. Among these, are reports about triglyceride hydrolysis [33], ester synthesis [34-37] and the preparation of polyesters $[38,39]$.

\subsection{Small Molecules}

The enzymatic hydrolysis of tricaprylin in miniemulsion was conducted with two different lipases: Lipase PS from Pseudomonas cepacia and lipase from Rhizopus arrhizus (RAL) [33]. Using enzyme concentrations of $5 \mathrm{mg} \mathrm{mL} \mathrm{m}^{-1}, 85 \%$ of the tricaprylin was hydrolyzed after $4 \mathrm{~h}$ with reaction temperatures of 35 or $40{ }^{\circ} \mathrm{C}$. The amounts of diglycerides and monoglycerides found in the product mixture changed with time and were dependent on the preferential hydrolysis site of the lipase. 
Interestingly, Lipase PS, an enzyme, which is known to be non-regiospecific in conventional emulsion [21,22], showed a distinct preference for hydrolysis in the central sn-2 position of tricaprylin (see Scheme 2).

This was manifested in the high concentration of 1,3-diglyceride of almost $20 \%$ in the reaction mixture after $24 \mathrm{~h}$. RAL, in contrast, did not show any altered selectivity. The preference for the sn-1,3 position, exhibited in conventional emulsion, was still observed in miniemulsion. In addition to hydrolysis, experiments on glycerolysis were conducted. In contrast to conventional glycerolysis, which is typically conducted with very low amounts of water, here, glycerol was added to the aqueous continuous phase. Pure glycerol could not be used for the generation of a miniemulsion, as the high viscosity of glycerol prevented the homogenization of the miniemulsion. In the experiments with RAL as catalyst, no significant changes in the product composition after $24 \mathrm{~h}$ were detected. The Lipase PS catalyzed glycerolysis, however, led to a significantly lower concentration of 1,3-diglyceride compared to the hydrolysis reaction. This indicates that the regioselectivity of Lipase PS is also changed by the presence of glycerol in the system. In the glycerolysis reaction, a preference for the sn-1,3 position is observed. These results are interesting, as the regioselectivity of an enzyme was changed by the physical environment (emulsion $\rightarrow$ miniemulsion), as well as by the addition of a reaction component, glycerol. The authors also showed that the addition of isophorone diisocyanate at different stages of the hydrolysis led to a polyurethane of different properties. These results show that lipase catalyzed hydrolysis of triglycerides is a potential way to produce raw materials for polymeric materials with a convenient and resource-saving process.

Lipase PS catalyzed hydrolysis was also used for the resolution of a racemic mixture of rac- $\beta$-phenylalanine $n$-propyl ester [40]. The reaction in miniemulsion was compared to the industrially used reaction in methyl-t-butyl ether/water mixtures. Both reactions proceeded to give about a $40 \%$ yield of the enantiomerically pure $S$ - $\beta$-phenylalanine. The final conversion of the reactant of about $50 \%$ was reached after $6 \mathrm{~h}$ compared to $15 \mathrm{~h}$ in the conventional system. The greatest benefit from the miniemulsion-based process, however, is the ability to increase the substrate concentration dramatically (up to $2.3 \mathrm{~mol} \mathrm{~L} \mathrm{~L}^{-1}$ ) without a significant drop of conversion. Due to the compartmentalization, the increased substrate load does not significantly affect the viscosity of the emulsion. Similar reactions were conducted with the n-propyl ester of rac- $\alpha$-phenylalanine. Here, substrate concentrations of up to $4 \mathrm{~mol} \mathrm{~L}^{-1}$ were handled without problems.

If the reaction system is properly chosen, not only hydrolysis, but also lipase catalyzed esterification can be conducted in aqueous emulsions [24]. Looking at the overall system, such reactions seem to be a contradiction to the reaction equation of dehydrative esterification from a carboxylic acid and an alcohol (see Scheme 1). In the presence of (large amounts of) water, the equilibrium is shifted to the reactant side.

As already mentioned above, Okumura et al. reported the lipase catalyzed synthesis of oleyl esters in aqueous medium, without giving a proper explanation to this contradiction. Baille et al. [41] used an emulsion approach for the chemically catalyzed polycondensation of diols with carboxylic diacids or dialdehydes to synthesize polyester and polyacetals, respectively. The authors found that the most efficient catalysts for polycondensation were sulfate-based and interfacially active. They concluded that the water removal from the reaction site is essential and speculated about cation- and surfactant-assisted mechanisms of water transport from the droplets [41]. Kobayashi et al. [42] and 
Suda et al. [43] reported on the dehydrative polycondensation of $\alpha, \omega$-dicarboxylic acids and several diols. Catalyzed by lipases of different origins, a variety of polyesters were synthesized, despite the presence of water in the reaction mixture. Manabe et al. investigated the esterification and other dehydrative reactions catalyzed by p-dodecylbenzenesulfonic acid, an acidic surfactant, in emulsions of carboxylic acids and alcohol. Their results showed that the reactants and the products have to be sufficiently hydrophobic to exclude water from the reaction site, and ideally, the catalyst can also act as a surfactant $[44,45]$.

Inspired by these results, a detailed basic study on the esterification of carboxylic acids and phenylalkanoles in miniemulsion was presented by Aschenbrenner et al. [34]. This study covers a variety of the aspects of lipase catalyzed esterification in miniemulsion. A crucial point for the creation of a suitable, stable miniemulsion is the choice of surfactant. Enzymes are influenced by the interaction with surfactants. Some interactions may increase the activity [28]; most enzymes, however, interfere with and reduce the enzyme activity. In the case of lipases, sodium dodecyl sulfate cannot be used, as it inhibits lipase catalysis. Ester-based surfactants, such as polysorbates, are prone to cleavage. Thus, poly(ethylene oxide)-based ethers were mainly used for stabilization. As a prerequisite for being able to conduct the reaction at only slightly ambient temperatures, the reactant mixture has to be liquid at the reaction temperature. This excludes acids with higher melting points than about $40{ }^{\circ} \mathrm{C}$. Systems with carboxylic acids from seven to 12 carbon atoms in the chain and phenylalkanoles from benzyl alcohol to 5-phenylpentanol were investigated. The authors showed that the stability of the miniemulsion during the reaction is mainly dependent on the water solubility of the carboxylic acid, but not on the water solubility of the alcohol. Miniemulsions with heptanoic acid were stable; systems with shorter chain acids were not sufficiently stable to conduct the esterification during $24 \mathrm{~h}$. Interestingly, the water solubility of the alcohol did not affect the stability of the miniemulsion. Even in reactions with highly water soluble benzyl alcohol (solubility in water: $0.37 \mathrm{~mol} \mathrm{~L}^{-1}$ ), stable miniemulsions were produced, and an efficient and quick reaction was observed $(80 \%$ conversion after $4 \mathrm{~h}, 5 \mathrm{mg} \mathrm{mL}^{-1}$ Lipase PS).

Figure 2 shows the temporal evolution of the conversion of the individual acids with 3-phenlypropanol and the activity of Lipase PS in the respective systems. Two effects seem to play a role: first, the selectivity of the enzyme towards an acid with a certain chain length (here, nonanoic acid) and, second, the hydrophobicity of reactants and products. In experiments with a combination of acids, it was shown that the hydrophobicity of the acid is a relevant factor and, in combination with the enzyme selectivity, is the decisive factor for the reaction rates. The reactions in miniemulsion were also compared to reactions conducted in bulk (here, no reaction was observed) and in conventional emulsion. The data showed that the conversion in a conventional, stirred emulsion is slower by about 50\%. A comparison between 4-dodecylbenzenesulfonic acid (DBSA) catalyzed esterification of nonanoic acid with 3-phenylpropanol with literature data [44] also underlines that the fine droplets and the high interfacial area of a miniemulsion accelerate the conversion when compared to a conventional emulsion. 
Figure 2. (a) Conversion versus time of the reaction of 3-phenylpropanol with linear carboxylic acids with chain lengths of $\mathrm{C} 7-\mathrm{C} 12$ in the presence of Lipase PS. The conversion data was obtained by HPLC analysis and confirmed with NMR spectroscopic analysis. The conversion rates were calculated from the initial slopes: C7: 0.10; C8: 0.15; C9: $0.32 ; \mathrm{C} 10: 0.22 ; \mathrm{C} 11: 0.10 ; \mathrm{C} 12: 0.21 \mathrm{~min}^{-1}$. The dotted lines are only a guide for the eye; (b) Enzyme activity of the reactions of 3-phenylpropanol with carboxylic acid substrates with increasing carbon-chain length. The values are calculated from the initial slopes obtained from the profiles in graph (a) [34].
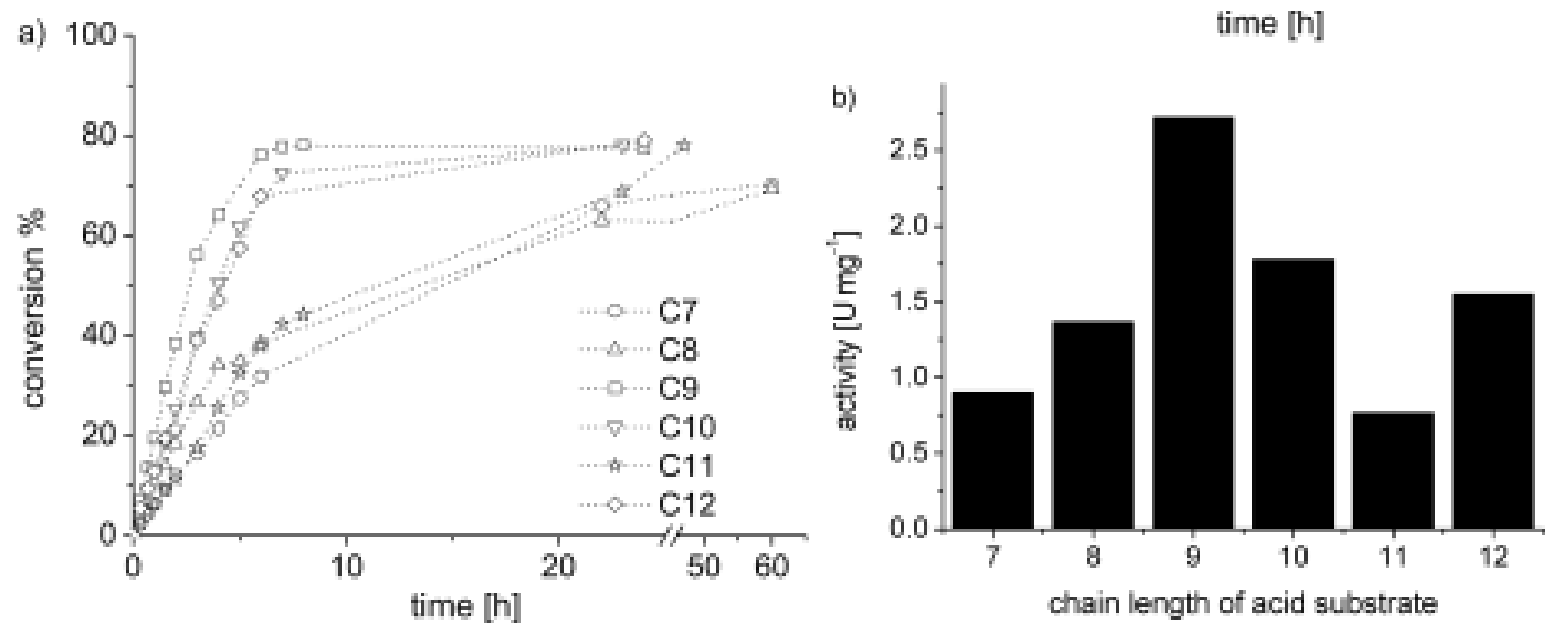

Figure 3. Esterification yields for ethyl (a) and hexyl (b) esters at $24 \mathrm{~h}$ in miniemulsion (ME 80\%,w/v) for three different enzymes: Amano Lipase PS, Candida rugosa lipase and Fusarium solani pisi cutinase [36].
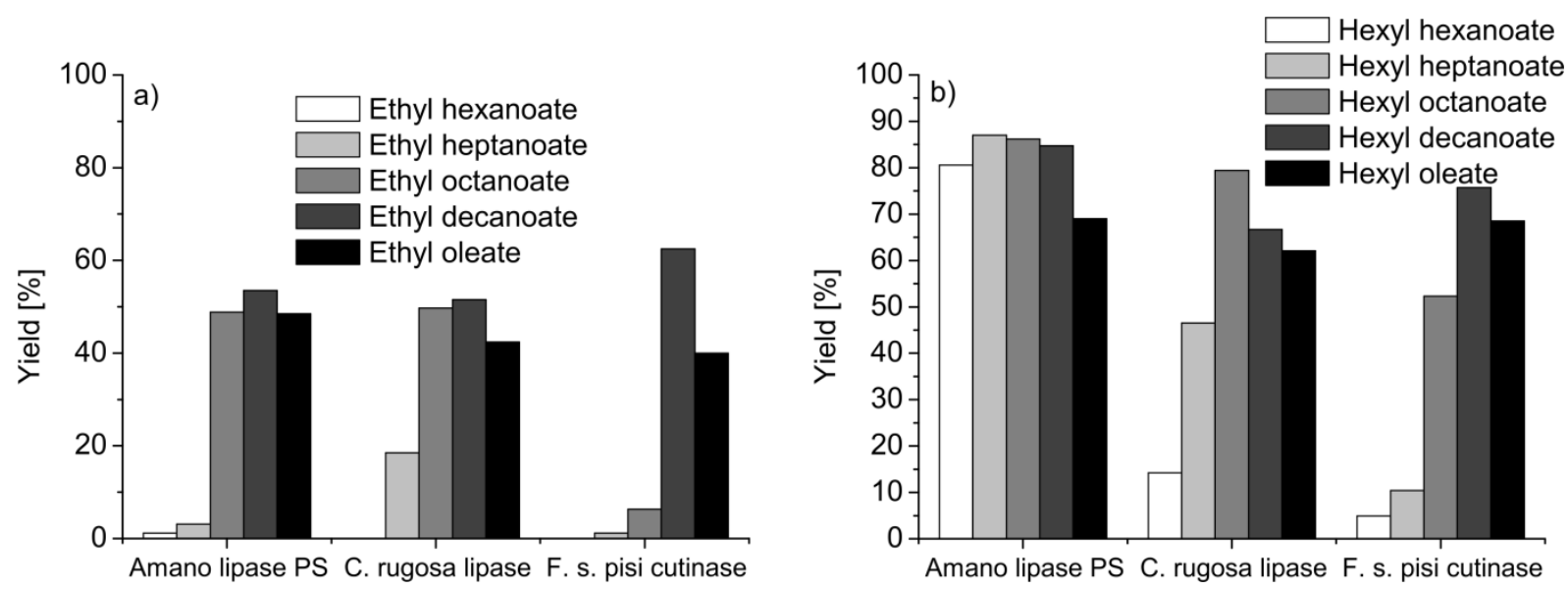

Enzymatic esterification in miniemulsion using lipases and cutinase, an enzyme that is functionally related to the lipases, but also able to work in the aqueous phase [30], was used in miniemulsion for the preparation of industrially relevant flavor esters (acids: C6-C10, C18; alcohols: C2-C6) [35-37,46]. When cutinase is used for esterification in organic solvents, such as, e.g., hexane [37] or iso-octane [47-49] or reverse micellar systems [50,51], a preference for short-chain carboxylic acids is observed, which is manifested in higher reaction rates and significant ester yields. In miniemulsion, only low yields of up to $20 \%$ of ester were obtained when ethanol was esterified with hexanoic or heptanoic acid, using 
Lipase PS, lipase from Candida rugosa or recombinant cutinase from Fusarium solani pisi. Using octanoic, decanoic or oleic acid, approximately a 50\% yield was obtained. Interestingly, the yield of the cutinase catalyzed reaction was highest for decanoic acid and almost insignificant for octanoic acid (Figure $3(\mathrm{a})$ ).

Using hexanol for the esterification, the equilibrium yields (after $24 \mathrm{~h}$ ) were generally higher than in the reactions with ethanol (Figure 3 (b)). In Lipase PS catalyzed reactions, almost all acids yielded more than $70 \%$ of ester; the highest yield in the reaction catalyzed with lipase from Candida rugosa was obtained with octanoic acid and with decanoic acid in the reaction catalyzed with cutinase. The position of the equilibrium of the reaction should be the same for each enzyme, as the enzyme only acts as a catalysis, accelerating the establishment of the equilibrium. The authors attributed this to a change in the microenvironment of the miniemulsion. The miniemulsions were stable with both alcohols; upon addition of the enzyme preparation, it was observed that the enzyme preparations have different tendencies to induce phase separation. If phase separation occurs, the high surface area provided in a stable miniemulsion is no longer available, which reduced the reaction rate significantly. Possible reasons may be the competition with the enzymes and the surfactant for the droplet interface [20], which lead to the destabilization of the miniemulsion. The authors also increased the substrate load in the miniemulsion from $20 \%$ to $50 \%$. Although the yields dropped, the overall product concentration was significantly increased (by a factor of about two).

Inhibition of the enzyme was observed in cutinase, as well as in Lipase PS catalyzed miniemulsions. Lipase PS was found to be inhibited by the presence of excessive acid [34]; cutinase was found to be inhibited by excesses of alcohol in the reaction mixture in miniemulsion or organic solvents [49] and by excessive acid in reverse micelles with cetyl trimethyl ammonium bromide (CTAB) [50]. The conformation of cutinase was found to be crucially dependent on the ratio of acid/alcohol in the miniemulsion droplet, which may be a reason for the inhibitory effect [35].

In addition to "conventional" substrates (acid and alcohol), lipases can also catalyze the amidation of an acid with an amine or ammonia [52,53]. Ragupathy et al. [54] investigated a system of hydrophobic amines and lactones and found that instead of the expected direct amidation of the lactone, first the lactone is hydrolyzed to the $\omega$-hydroxycarboxylic acid and then amidation takes place (see Figure 4).

Detailed investigations showed that at low enzyme concentrations (Lipase PS, $7 \mathrm{mg} \mathrm{mL}^{-1}$ ), the hydrolysis of the lactone is the major reaction during the first $24 \mathrm{~h}$, whereas with increasing enzyme concentrations, the conversion to the amide product also increases. Longer reaction times also favored the production of the amide. Pentadecanolide (PDL) was completely hydrolyzed to 15-hydroxypentadecanoic acid within $30 \mathrm{~min}$ using enzyme concentrations of $67 \mathrm{mg} \mathrm{L}{ }^{-1}$. This, in combination with directly using 15-hydroxypentadecanoic acid as a substrate, led to the conclusion that not the lactone, but the $\omega$-hydroxy acid is the substrate for amidation. The amidation reaction, however, needed about one week to reach $90 \%$ conversion. This long reaction time was ascribed to the formation of a salt from the amine and carboxylic acid. In the protonated and deprotonated state, the reactants are not suitable for enzymatic amidation. In the equilibrium state, a small amount of the acid and amine is present, which can then react to the amide. Without lipase, no reaction takes place. With optimized reaction conditions, 94\% of amide (from hexadecanolide or pentadecanolide and oleylamine) could be observed. 
Figure 4. Novel reaction pathway for aminolysis of a lactone demonstrated by ${ }^{1} \mathrm{H}$ NMR analysis. Conversions are obtained from kinetic study between oleylamine and pentadecanolide. Reprinted from [54]. Copyright (2000), with permission from Elsevier.

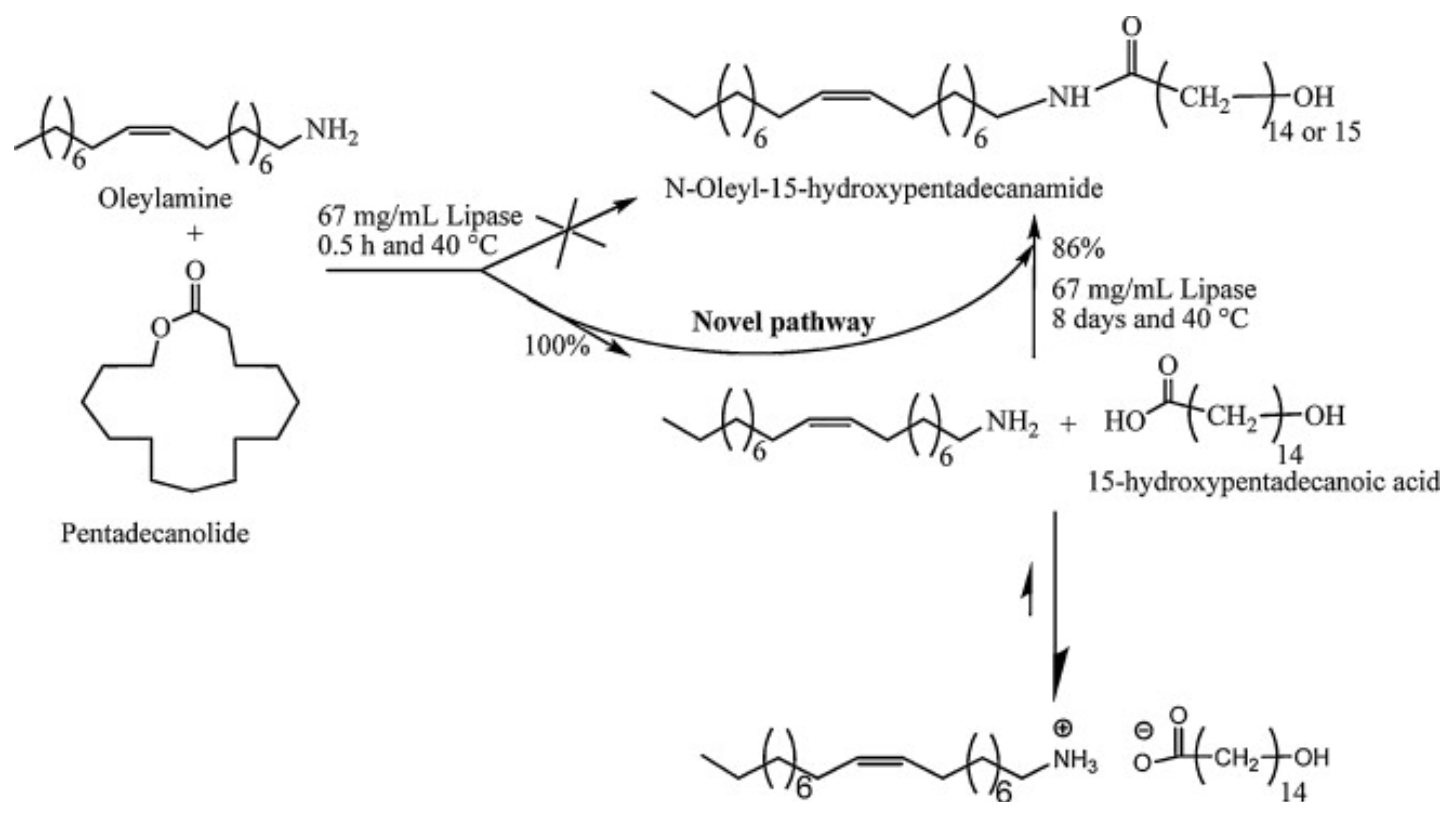

\subsection{Polymers}

Enzymes have been used for polymerization reactions since the late 80 s of the last century (see, e.g., [55-57] and the literature therein). Using lipases for synthesis is a very well-studied system, and being a biodegradable and biotolerable polymer, the polyesters have found wide application in, e.g., biomedicine. Two general mechanisms were identified for lipase catalyzed synthesis of polyesters: (1) the ring opening polymerization (ROP) of lactones and (2) the polycondensation of carboxylic acids/esters and alcohols or the polycondensation of hydroxycarboxylic acids/esters (Figure 5). A detailed description of the mechanisms is beyond the scope of this review and can be found elsewhere [57,58].

Figure 5. Two major modes of lipase-catalyzed polyester synthesis [56].

(1) Ring-Opening Polymerization of Lactones

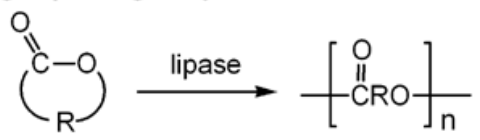

(2) Polycondensation

(a) Carboxylic Acids or Their Esters with Alcohols

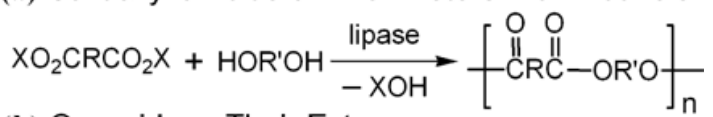

(b) Oxyacids or Their Esters

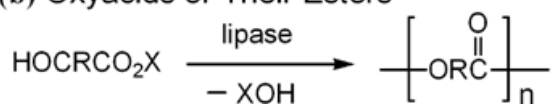

Despite the environmental implications of water-based systems and the fact that a lipase can act in its natural, aqueous environment, only a few reports about the use of a miniemulsion for the synthesis of polyesters can be found in the literature covering the enzymatic polymerization of lactones, i.e., 
pentadecanolide (PDL) and hexadecanolide (HDL). As the melting point of PDL is around $35{ }^{\circ} \mathrm{C}$, the reactions were conducted at slightly elevated temperatures of 40 [38] or $45{ }^{\circ} \mathrm{C}$ [39]. The data of Målberg et al. [38] indicate full conversion of PDL and HDL to polymer after $5 \mathrm{~h}$ of reaction (2\% Lipase PS, $40{ }^{\circ} \mathrm{C}$ ). Although a direct comparison is difficult, as the protein contents and the activities of the enzymes may vary, the value agrees with the data of Taden et al. [39], who observed full conversion after $4-4.5 \mathrm{~h}$ using $0.5 \%$ of Lipase PS. These data show the superiority of conducting the reaction in miniemulsion in comparison to other reaction systems. In neat lactone, Målberg et al. did not observe any conversion using Lipase PS and only conversions of $20 \%$ within $3.5 \mathrm{~h}$ using Lipase AK (from Pseudomonas fluorescens); reactions in organic solvents need higher amounts of enzyme and require longer reaction times [58]. The enzyme seems to work more efficiently in its native, aqueous environment. The molecular weights obtained by Målberg et al. were typically $1200 \mathrm{~g} \mathrm{~mol}^{-1}<M_{n}<2300 \mathrm{~g} \mathrm{~mol}^{-1}$; however, one experiment led to a polymer with $M_{n}=9300 \mathrm{~g} \mathrm{~mol}^{-1}$ (all determined with size exclusion chromatography (SEC) against PS standards). These data are in agreement with the molecular weights of polyesters obtained from the ROP of PDL synthesized in dispersion [59-61]. In contrast, the molecular weights observed by Taden et al. are extraordinarily high being, more than $200,000 \mathrm{~g} \mathrm{~mol}^{-1}$. Attempting to understand the high molecular weight, the authors investigated the importance and the relative velocities of the possible reactions (ROP and polycondensation). A combination of ROP and polycondensation was reported to be responsible for the high molecular weight. Systematic studies, however, are still required in order to fully understand the process and to evaluate the parameters to increase the molecular weight of the polyesters. With this achieved, the process may become relevant for the production of application-relevant polyesters. One of the advantages to underline is that conducting the reaction in miniemulsion directly leads to nano-sized particles the size of a few hundreds of nanometers, dispersed in water.

So far, only these lactones have successfully been used for lipase catalyzed synthesis of polyesters in miniemulsion. Attempts to use L-lactide (LLA) for the lipase catalyzed synthesis of LLA in miniemulsion were so far not successful [38]. This reaction, however, could be conducted in bulk LLA with ethylene glycol as initiator. The reaction temperature of $100-120^{\circ} \mathrm{C}$ underlines the wide range of temperatures in which lipases can work.

\section{Enzyme Cleavable Nanostructures}

Miniemulsions are not only a suitable and efficient reaction environment for conducting enzyme catalyzed reactions, miniemulsions can also be used as a platform for the defined synthesis of functionally responsive nanomaterials. In addition to hydrolytically degradable polyesters or esters of polyacrylates, polymethacrylates and, especially, polycyanoacrylates, which are prone to chemical and enzymatically catalyzed hydrolysis [62-65], distinct and defined enzyme responsive/cleavable structures could be integrated in nanoparticles and nanocapsules. These structures can be, among others, polysaccharides [66,67] and peptides [68,69].

Klinger et al. [66,67] based his systems on modified dextrans, which are degraded by the corresponding enzyme dextranase. Dextran was esterified with methacrylic anhydride in different degrees of substitution. This was used as an enzyme degradable cross-linker in poly(acrylamide) 
hydrogels prepared in inverse miniemulsion. Additionally, a photocleavable cross-linker was introduced in this system. Thus, dual responsive nanoparticles were created. The swelling and degradation behavior is dependent on the amount of cross-linker added to the hydrogels and on the degree of substitution with methacroyl residues.

Peptide sequences, cleaved by specific enzymes, were integrated in polymeric particles [69], as well as in the shells of polymeric nanocapsules [68]. The trypsin-cleavable sequence, glycine-glycine-phenylalanine (GFF), was thus used as a cross-linker for polystyrene-co-poly(acrylic acid) (PS-co-PAA). For the preparation of nanocapsules, an additional reaction between the peptide (soluble in the droplet phase) and a diisocyanate (soluble in the continuous phase) at the interface of inverse miniemulsions led to the formation of the enzyme-cleavable hybrid capsules. For optical monitoring of the enzymatic cleavage, the peptide sequence was framed by an amino acid-based fluorophore-quencher pair (internally quenched fluorescence, IQF). In the intact state, the fluorescence intensity is low; however, upon cleavage, the intensity is increased. The crosslinking step was performed in inverse miniemulsion and led to nanostructures with the size of $100 \mathrm{~nm}$. Incubating the particles with trypsin led to a recovery of the fluorescence of the fluorophore and allowed detection of the enzyme in very low concentrations. The same IQF-peptide sequence was used for a polycondensation reaction at the interface of droplets in inverse miniemulsion [68]. This procedure could be used to achieve nanocapsules with a hydrophilic liquid core containing the fluorescent dye, sulforhodamine 101, in self-quenching concentrations. Incubation of the capsules with trypsin led to a recovery of the fluorescence of the fluorophore of the IQF peptide, as well as of the sulforhodamine 101, indicating that the capsule shell is cleaved and, simultaneously, the interior is liberated to the surrounding medium.

\section{Concluding Remarks}

We have presented an overview of how the miniemulsion technique can act as a versatile reaction medium for an enzyme catalyzed reaction. Especially lipases, which catalyze reactions at the interface between the hydrophobic substrates and the aqueous surrounding, benefit from the huge interface provided in miniemulsions. The results are quick esterification reactions from carboxylic acids with alcohols at only slightly elevated temperatures. The technique allows processing and synthesizing of small molecules, as well as macromolecules. We are convinced that in the future, further enzymes will be used for catalyzing reactions in miniemulsion and that such processes may become industrially relevant.

\section{Conflict of Interest}

The authors declare no conflict of interest.

\section{References}

1. Beloqui, A.; de María, P.D.; Golyshin, P.N.; Ferrer, M. Recent trends in industrial microbiology. Curr. Opin. Microbiol. 2008, 11, 240-248.

2. Nestl, B.M.; Nebel, B.A.; Hauer, B. Recent progress in industrial biocatalysis. Curr. Opin. Chem. Biol. 2011, 15, 187-193. 
3. Clouthier, C.M.; Pelletier, J.N. Expanding the organic toolbox: A guide to integrating biocatalysis in synthesis. Chem. Soc. Rev. 2012, 41, 1585-1605.

4. Bornscheuer, U.T.; Huisman, G.W.; Kazlauskas, R.J.; Lutz, S.; Moore, J.C.; Robins, K. Engineering the third wave of biocatalysis. Nature 2012, 485, 185-194.

5. Illanes, A.; Cauerhff, A.; Wilson, L.; Castro, G.R. Recent trends in biocatalysis engineering. Bioresour. Technol. 2012, 115, 48-57.

6. Simon, M.-O.; Li, C.-J. Green chemistry oriented organic synthesis in water. Chem. Soc. Rev. 2012, 41, 1415-1427.

7. Landfester, K. Synthesis of colloidal particles in miniemulsions. Annu. Rev. Mater. Res. 2006, 36, 231-279.

8. Landfester, K. Miniemulsion polymerization and the structure of polymer and hybrid nanoparticles. Angew. Chem. Int. Ed. 2009, 48, 4488-4507.

9. Landfester, K.; Weiss, C.K. Encapsulation by Miniemulsion Polymerization. Adv. Polym. Sci. 2010, 229, 1-49.

10. Weiss, C.K.; Landfester, K. Miniemulsion Polymerization as a Means to Encapsulate Organic and Inorganic Materials. Adv. Polym. Sci. 2010, 233, 185-236.

11. Munoz-Espi, R.; Weiss, C.K.; Landfester, K. Inorganic nanoparticles prepared in miniemulsion. Curr. Opin. Colloid Interface Sci. 2012, 17, 212-224.

12. Holmberg, K. Organic and bioorganic reactions in microemulsions. Adv. Colloid Interface Sci. 1994, 51, 137-174.

13. Derango, R.A.; Chiang, L.C.; Dowbenko, R.; Lasch, J.G. Enzyme-mediated polymerization of acrylic monomers. Biotechnol. Tech. 1992, 6, 523-526.

14. Kalra, B.; Gross, R.A. Horseradish peroxidase mediated free radical polymerization of methyl methacrylate. Biomacromolecules 2000, 1, 501-505.

15. Singh, A.; Ma, D.; Kaplan, D.L. Enzyme-Mediated free radical polymerization of styrene. Biomacromolecules 2000, 1, 592-596.

16. Qi, G.; Jones, C.W.; Schork, F.J. Enzyme-initiated miniemulsion polymerization. Biomacromolecules 2006, 7, 2927-2930.

17. Kohri, M.; Kobayashi, A.; Fukushima, H.; Kojima, T.; Taniguchi, T.; Saito, K.; Nakahira, T. Enzymatic miniemulsion polymerization of styrene with a polymerizable surfactant. Polym. Chem. 2012, 3, 900-906.

18. Kohri, M.; Kobayashi, A.; Fukushima, H.; Taniguchi, T.; Nakahira, T. Effect of surfactant type on enzymatic miniemulsion polymerization using horseradish peroxidase as a catalyst. Chem. Lett. 2012, 41, 1131-1133.

19. Bechthold, N.; Landfester, K. Kinetics of miniemulsion polymerization as revealed by calorimetry. Macromolecules 2000, 33, 4682-4689.

20. Reis, P.; Holmberg, K.; Watzke, H.; Leser, M.E.; Miller, R. Lipases at interfaces: A review. Adv. Colloid Interface Sci. 2009, 147-148, 237-250.

21. Rogalska, E.; Cudrey, C.; Ferrato, F.; Verger, R. Stereoselective hydrolysis of triglycerides by animal and microbial lipases. Chirality 1993, 5, 24-30.

22. Schmid, R.D.; Verger, R. Lipases: Interfacial enzymes with attractive applications. Angew. Chem. Int. Ed. 1998, 37, 1609-1633. 
23. Wilde, P.J.; Chu, B.S. Interfacial colloidal aspects of lipid digestion. Adv. Colloid Interface Sci. 2011, 165, 14-22.

24. Okumura, S.; Iwai, M.; Tsujisaka, Y. Synthesis of various kinds of esters by 4 microbial lipases. Biochim. Biophys. Acta 1979, 575, 156-165.

25. Eggers, D.K.; Blanch, H.W.; Prausnitz, J.M. Extractive catalysis: Solvent effects on equilibria of enzymatic reactions in two-phase systems. Enzyme Microb. Technol. 1989, 11, 84-89.

26. Bornscheuer, U.T. Lipase-catalyzed syntheses of monoacylglycerols. Enzyme Microb. Technol. 1995, 17, 578-586.

27. Castro, G.R.; Knubovets, T. Homogeneous biocatalysis in organic solvents and water-organic mixtures. Crit. Rev. Biotechnol. 2003, 23, 195-231.

28. Biasutti, M.A.; Abuin, E.B.; Silber, J.J.; Correa, N.M.; Lissi, E.A. Kinetics of reactions catalyzed by enzymes in solutions of surfactants. Adv. Colloid Interface Sci. 2008, 136, 1-24.

29. Monot, F.; Borzeix, F.; Bardin, M.; Vandecasteele, J.-P. Enzymatic esterification in organic media: Role of water and organic solvent in kinetics and yield of butyl butyrate synthesis. Appl Microb. Biotechnol. 1991, 35, 759-765.

30. Carvalho, C.M.L.; Aires-Barros, M.R.; Cabral, J.M.S. Cutinase: From molecular level to bioprocess development. Biotechnol. Bioeng. 1999, 66, 17-34.

31. Rona, P.; Ammon, R. Tests on enzymatic ester-hydrolysis and ester-synthesis. Biochem. Z. 1932, 249, 446-454.

32. Sym, E.A. On the esterase-effect. III. Biochem. Z. 1933, 258, 304-324.

33. de Lima, A.P.D.; Aschenbrenner, E.M.; Oliveira, S.; Doucet, J.-B.; Weiss, C.K.; Ziener, U.; Fonseca, L.P.; Ricardo, N.M.P.S.; de Freitas, L.L.; Petzhold, C.L.; et al. Towards regioselective enzymatic hydrolysis and glycerolysis of tricaprylin in miniemulsion and the direct preparation of polyurethane from the hydrolysis products. J. Mol. Catal., to be submitted for publication, 2013.

34. Aschenbrenner, E.M.; Weiss, C.K.; Landfester, K. Enzymatic esterification in aqueous miniemulsions. Chem. Eur. J. 2009, 15, 2434-2444.

35. de Barros, D.P.C.; Fernandes, P.; Cabral, J.M.S.; Fonseca, L.P. Synthetic application and activity of cutinase in an aqueous, miniemulsion model system: Hexyl octanoate synthesis. Catal. Today 2011, 173, 95-102.

36. de Barros, D.P.C.; Fonseca, L.P.; Cabral, J.M.S.; Aschenbrenner, E.M.; Weiss, C.K.; Landfester, K. Miniemulsion as efficient system for enzymatic synthesis of acid alkyl esters. Biotechnol. Bioeng. 2010, 106, 507-515.

37. de Barros, D.P.C.; Fonseca, L.P.; Cabral, J.M.S.; Weiss, C.K.; Landfester, K. Synthesis of alkyl esters by cutinase in miniemulsion and organic solvent media. Biotechnol. J. 2009, 4, 674-683.

38. Målberg, S.; Finne-Wistrand, A.; Albertsson, A.-C. The environmental influence in enzymatic polymerization of aliphatic polyesters in bulk and aqueous mini-emulsion. Polymer 2010, 51, 5318-5322.

39. Taden, A.; Antonietti, M.; Landfester, K. Enzymatic polymerization towards biodegradable polyester nanoparticles. Macromol. Rapid Commun. 2003, 24, 512-516.

40. Groger, H.; May, O.; Husken, H.; Georgeon, S.; Drauz, K.; Landfester, K. Enantioselective enzymatic reactions in miniemulsions as efficient "nanoreactors". Angew. Chem. Int. Ed. 2006, $45,1645-1648$. 
41. Baile, M.; Chou, Y.J.; Saam, J.C. Direct polyesterification in aqueous emulsion. Polym. Bull. 1990, 23, 251-257.

42. Kobayashi, S.; Uyama, H.; Suda, S.; Namekawa, S. Dehydration polymerization in aqueous medium catalyzed by lipase. Chem. Lett. 1997, 26, doi:10.1246/cl.1997.105.

43. Suda, S.; Uyama, H.; Kobayashi, S. Dehydration polycondensation in water for synthesis of polyesters by lipase catalyst. Proc. Jpn. Acad. Ser. B 1999, 75, 201-206.

44. Manabe, K.; Iimura, S.; Sun, X.M.; Kobayashi, S. Dehydration reactions in water. Bronsted acid-surfactant-combined catalyst for ester, ether, thioether, and dithioacetal formation in water. J. Am. Chem. Soc. 2002, 124, 11971-11978.

45. Manabe, K.; Sun, X.M.; Kobayashi, S. Dehydration reactions in water. Surfactant-type bronsted acid-catalyzed direct esterification of carboxylic acids with alcohols in an emulsion system. J. Am. Chem. Soc. 2001, 123, 10101-10102.

46. De Barros, D.P.C.; Fonseca, L.P.; Cabral, J.M.S.; Weiss, C.K.; Landfester, K. Biosynthesis of fatty acids alkyl esters in miniemulsion as a reaction media. New Biotechnol. 2009, 25, S116.

47. de Barros, D.P.C.; Fernandes, P.; Cabral, J.M.S.; Fonseca, L.P. Operational stability of cutinase in organic solvent system: Model esterification of alkyl esters. J. Chem. Technol. Biotechnol. 2010, $85,1553-1560$.

48. De Barros, D.P.C.; Azevedo, A.M.; Cabral, J.M.S.; Fonseca, L.P. Optimization of flavor esters synthesis by fusarium solani pisi cutinase. J. Food Biochem. 2012, 36, 275-284.

49. de Barros, D.P.C.; Fonseca, L.P.; Fernandes, P.; Cabral, J.M.S.; Mojovic, L. Biosynthesis of ethyl caproate and other short ethyl esters catalyzed by cutinase in organic solvent. J. Mol. Catal. B 2009, 60, 178-185.

50. Cunnah, P.J.; Aires-Barros, M.R.; Cabral, J.M.S. Esterification and transesterification catalysed by cutinase in reverse micelles of ctab for the synthesis of short chain esters. Biocatal. Biotransform. 1996, 14, 125-146.

51. Pinto-Sousa, A.M.C.; Cabral, J.M.S.; Aires-Barros, M.R. Ester Synthesis by a recombinant cutinase in reversed micelles of a natural phospholipid. Biocatal. Biotransform. 1994, 9, 169-179.

52. Gotor, V. Non-conventional hydrolase chemistry: Amide and carbamate bond formation catalyzed by lipases. Biorg. Med. Chem. 1999, 7, 2189-2197.

53. Gotor-Fernández, V.; Busto, E.; Gotor, V. Candida antarctica lipase b: An ideal biocatalyst for the preparation of nitrogenated organic compounds. Adv. Synth. Catal. 2006, 348, 797-812.

54. Ragupathy, L.; Pluhar, B.; Ziener, U.; Keller, H.; Dyllick-Brenzinger, R.; Landfester, K. Enzymatic aminolysis of lactones in aqueous miniemulsion: Catalysis through a novel pathway. J. Mol. Catal. B 2010, 62, 270-276.

55. Gross, R.A.; Ganesh, M.; Lu, W. Enzyme-catalysis breathes new life into polyester condensation polymerizations. Trends Biotechnol. 2010, 28, 435-443.

56. Kobayashi, S. Recent developments in lipase-catalyzed synthesis of polyesters. Macromol. Rapid Commun. 2009, 30, 237-266.

57. Kobayashi, S.; Makino, A. Enzymatic polymer synthesis: an opportunity for green polymer chemistry. Chem. Rev. 2009, 109, 5288-5353.

58. Kobayashi, S.; Uyama, H.; Kimura, S. Enzymatic polymerization. Chem. Rev. 2001, 101, 3793-3818. 
59. Namekawa, S.; Uyama, H.; Kobayashi, S. Lipase-catalyzed ring-opening polymerization of lactones in water. Polym. J. 1998, 30, 269-271.

60. Nallani, M.; de Hoog, H.-P.M.; Cornelissen, J.J.L.M.; Palmans, A.R.A.; van Hest, J.C.M.; Nolte, R.J.M. Polymersome nanoreactors for enzymatic ring-opening polymerization. Biomacromolecules 2007, 8, 3723-3728.

61. Panlawan, P.; Luangthongkam, P.; Wiemann, L.O.; Sieber, V.; Marie, E.; Durand, A.; Inprakhon, P. Lipase-catalyzed interfacial polymerization of $\omega$-pentadecalactone in aqueous biphasic medium: A mechanistic study. J. Mol. Catal. B 2013, 88, 69-76.

62. Gardella, J.A.; Novak, F.P.; Hercules, D.M. Static secondary ion mass-spectrometry for study of surface hydrolysis of poly(tert-butyl methacrylate). Anal. Chem. 1984, 56, 1371-1375.

63. O'Sullivan, C.; Birkinshaw, C. Hydrolysis of poly (n-butylcyanoacrylate) nanoparticles using esterase. Polym. Degrad. Stable 2002, 78, 7-15.

64. Sullivan, C.O.; Birkinshaw, C. In vitro degradation of insulin-loaded poly (n-butylcyanoacrylate) nanoparticles. Biomaterials 2004, 25, 4375-4382.

65. Vauthier, C.; Dubernet, C.; Fattal, E.; Pinto-Alphandary, H.; Couvreur, P. Poly(alkylcyanoacrylates) as biodegradable materials for biomedical applications. Adv. Drug Deliv. Rev. 2003, 55, 519-548.

66. Klinger, D.; Aschenbrenner, E.M.; Weiss, C.K.; Landfester, K. Enzymatically degradable nanogels by inverse miniemulsion copolymerization of acrylamide with dextran methacrylates as crosslinkers. Polym. Chem. 2012, 3, 204-216.

67. Klinger, D.; Landfester, K. Enzymatic- and light-degradable hybrid nanogels: Crosslinking of polyacrylamide with acrylate-functionalized dextrans containing photocleavable linkers. J. Polym. Sci. Part A 2012, 50, 1062-1075.

68. Andrieu, J.; Kotman, N.; Maier, M.; Mailander, V.; Strauss, W.S.L.; Weiss, C.K.; Landfester, K. Live monitoring of cargo release from peptide-based hybrid nanocapsules induced by enzyme cleavage. Macromol. Rapid Commun. 2012, 33, 248-253.

69. Maier, M.; Kotman, N.; Friedrichs, C.; Andrieu, J.; Wagner, M.; Graf, R.; Strauss, W.S.L.; Mailander, V.; Weiss, C.K.; Landfester, K. Highly site specific, protease cleavable, hydrophobic peptide-polymer nanoparticles. Macromolecules 2011, 44, 6258-6267.

(C) 2013 by the authors; licensee MDPI, Basel, Switzerland. This article is an open access article distributed under the terms and conditions of the Creative Commons Attribution license (http://creativecommons.org/licenses/by/3.0/). 